\title{
Organic molecules in meteorites
}

\author{
Zita Martins \\ Dept of Earth Science and Engineering, Imperial College London, South Kensington Campus, \\ London SW7 2AZ, UK \\ email: z.martins@imperial.ac.uk
}

\begin{abstract}
The analysis of the organic content of meteorites provides a window into the conditions of the early solar system, such as the extension of aqueous alteration or thermal metamorphism on the meteorite parent bodies. The analysis of the soluble organic content of CM chondrites indicates that extensive aqueous alteration on their meteorite parent body may result on 1) the decomposition of $\alpha$-amino acids; 2) synthesis of $\beta$ - and $\gamma$-amino acids; 3 ) higher relative abundances of alkylated polycyclic aromatic hydrocarbons (PAHs); and 4) higher L-enantiomer excess (Lee) value of isovaline. Exogenous delivery of organic matter by meteorites may have contributed to the organic inventory of the early Earth, providing a diversity of resources to the first living organisms on Earth and on other places of our solar system where life could have potentially originated.
\end{abstract}

\section{Introduction}

Carbonaceous meteorites are primitive samples from the asteroid belt, containing 3$5 \mathrm{wt} \%$ organic carbon. The exogenous delivery of organic matter by carbonaceous meteorites may have contributed to the organic inventory of the early Earth, as the inner solar system was heavily bombarded from around 4.5 to 3.8 billion years ago (Schidlowski 1988, Schopf 1993, Chyba \& Sagan 1992). The majority (>70\%) of the meteoritic organic material consist of insoluble organic matter (IOM) (Cody and Alexander 2005), while the remaining meteoritic organic material $(<30 \%)$ consists of a rich organic inventory of soluble organic compounds, including key compounds important in terrestrial biochemistry (Cronin \& Chang 1993, Martins \& Sephton 2009, Martins 2011). Bulk analysis of the meteoritic soluble organic fraction has revealed a high molecular diversity of tens of thousands of different molecular compositions (Schmitt-Kopplin et al. 2010).

Different carbonaceous meteorites contain soluble organic molecules with different abundances and distributions, which may reflect the extension of aqueous alteration or thermal metamorphism on the meteorite parent bodies. For example, the amino acid contents of the CO3s Colony and Ornans were low but contained unusually high relative abundances of $\beta$-alanine and $\gamma$-ABA (Chan et al. 2012). This may be explained by amino acids formation by Fischer-Tropsch / Haber-Bosch type gas-grain reactions after the meteorite parent body cooled to much lower temperatures (Glavin et al. 2010, Burton et al. 2011) or during the cooling process in the parent body, still at elevated temperatures but lower than $500{ }^{\circ} \mathrm{C}$ (Rodante et al. 1992).

Aqueous alteration on the meteorite parent body of carbonaceous chondrites contributes to the alteration of their mineral, isotopic and volatile content (e.g. Clayton \& Mayeda 1984, Tomeoka \& Buseck 1985, Zolensky \& McSween 1988,Zolensky et al. 1997, Browning et al. 1996, Palmer and Lauretta 2011). In addition, the relative distribution of amino acids in carbonaceous chondrites seems to be influenced by the degree of aqueous alteration on the parent body (Glavin et al. 2006, 2011, Pizzarello et al. 2008, Martins et al. 2007). To further test the influence of aqueous alteration on the soluble organic 


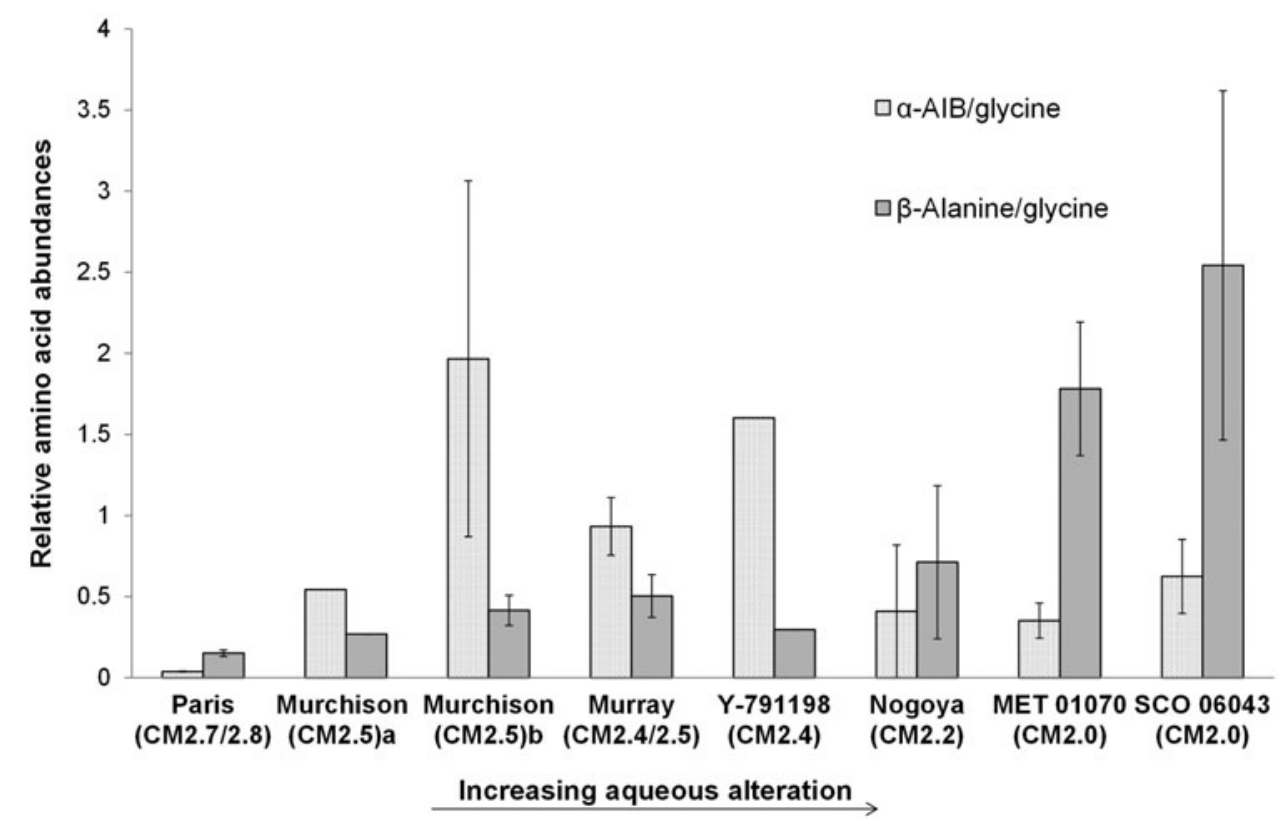

Figure 1. Relative abundances of $\alpha$-AIB/glycine (white) and $\beta$-alanine/glycine (grey) for CM chondrites with different degrees of aqueous alteration according to the hydrothermal scale designated by Rubin et al. (2007). Meteorites include the CM2.7/2.8 Paris (Martins et al. 2015), the CM2.5 Murchison (a) from Shimoyama and Ogasawara 2002; b) from Glavin et al. (2011), the CM2.4/2.5 Murray (Ehrenfreund et al. 2001, Botta et al. 2002), the CM2.4 Y-791198 (Shimoyama and Ogasawara (2002), the CM2.2 Nogoya (Botta et al. 2002), the CM2.0 MET 01070, and the CM2.0 SCO 06043 (Glavin et al. 2011).

content of carbonaceous meteorites, the amino acid and hydrocarbon contents of one of the least aqueously altered CM chondrites have been analysed (Martins et al. 2015).

\section{Aqueous alteration and the Paris meteorite}

The Paris meteorite is reported to be the a CM2.7 or CM2.8 on the hydrothermal scale designated by Rubin et al. (2007), i.e. one of the least altered CM chondrites (Blanchard et al. 2011, Bourot-Denise et al. 2010, Merouane et al. 2011, Caillet Komorowski et al. 2011, Cournede et al. 2011, Kimura et al. 2011). In addition, the IR spectra of Paris points to a primitive origin for the organic matter in this meteorite (Merouane et al. 2012).

The analysis of the amino acid content of Paris shows that the degree of aqueous alteration may be responsible for the amino acid abundances and distribution to some extent:

(a) Isovaline detected on the Paris meteorite was racemic (corrected $D / L=1.03$; corrected L-enantiomer excess $(\%)=-1.4 \pm 2.6$ ) (Martins et al. 2015). This supports the hypothesis that aqueous alteration is responsible for the high L-enantiomer excess of isovaline observed in the most aqueously altered carbonaceous meteorites. To note that aqueous alteration does not create by itself an isovaline asymmetry, but it may amplify a small enantiomeric excess.

(b) The least and medium aqueously altered CM chondrites (i.e. Paris, Murchison, Murray and Y-791198) have much higher relative abundances of 4-carbon $\alpha$-amino acids than $\beta$ - and $\gamma$-amino acids, when com-pared to the most aqueously altered CM chondrites. 
Table 1. Summary of the average total aliphatic ( $n$-alkanes) hydrocarbon abundances (in $\mathrm{ppb}$ ) in the Paris meteorite measured by GC-MS (Martins et al. 2015) ${ }^{a}$

\begin{tabular}{|c|c|}
\hline Aliphatic Hydrocarbons & |Concentration (ppb) \\
\hline $\mathrm{C}_{16} \mathrm{H}_{34}$ & $697 \pm 30$ \\
\hline $\mathrm{C}_{17} \mathrm{H}_{36}$ & $2499 \pm 230$ \\
\hline $\mathrm{C}_{18} \mathrm{H}_{38}$ & $1799 \pm 91$ \\
\hline $\mathrm{C}_{19} \mathrm{H}_{40}$ & $722 \pm 38$ \\
\hline $\mathrm{C}_{20} \mathrm{H}_{42}$ & $449 \pm 22$ \\
\hline $\mathrm{C}_{21} \mathrm{H}_{44}$ & $406 \pm 24$ \\
\hline $\mathrm{C}_{22} \mathrm{H}_{46}$ & $379 \pm 25$ \\
\hline $\mathrm{C}_{23} \mathrm{H}_{48}$ & $311 \pm 19$ \\
\hline $\mathrm{C}_{24} \mathrm{H}_{50}$ & $239 \pm 16$ \\
\hline $\mathrm{C}_{25} \mathrm{H}_{52}$ & $170 \pm 14$ \\
\hline Total & 7,670 \\
\hline
\end{tabular}

Notes:

${ }^{a}$ The associated errors are based on the standard deviation of the average value between six separate measurements (N) with a standard error, $\delta_{x}=\sigma x . N^{-1 / 2}$

The Strecker-cyanohydrin amino acid synthesis may have been more active in the least and medium aqueously altered CM chondrite.

(c) Extensive aqueous alteration in the parent body of carbonaceous meteorites results in the decomposi-tion of $\alpha$-amino acids (Botta et al. 2007, Martins et al. 2007). This is explained by increasing low-temperature chemical oxidation, which removes the aliphatic portions of the soluble and macromolecular organic material (Cody and Alexander 2005, Martins et al. 2006).

(d) Extensive aqueous alteration in the parent body of carbonaceous meteorites results in the synthesis of $\beta$ - and $\gamma$-amino acids. This may occur via the hydrolysis of lactams leading to the synthesis of $\beta$ - and $\gamma$-amino acids (Cooper and Cronin 1995), or by the Michael addition of ammonia to $\alpha, \beta$-unsaturated nitriles (forming $\beta$-amino alkylnitriles) followed by hydrolysis to form ]-amino acids (Cronin and Chang 1993).

$(e)$ The relative abundance of $\beta$-alanine (glycine $=1$ ) increases with increasing aqueous alteration. The Paris meteorite has the lowest relative abundance of $\beta$-alanine/glycine for a CM chondrite (0.15) (Figure 1), which fits with the relative abundance of $\beta$ alanine/glycine increasing with increasing aqueous alteration (Glavin et al. 2006, 2011).

The analysis of the hydrocarbon content of the Paris meteorite showed aliphatic hydrocarbons ( $n$-alkanes) ranging from $\mathrm{C}_{16}$ to $\mathrm{C}_{25}$ (Table 1 ), with no even or odd number predominance. It also contained 3- to 5-ring non-alkylated polycyclic aromatic hydrocarbons (PAHs). The Paris meteorite did not contain any alkylated PAHs, while alkylated PAHs species were detected in Murchison and Y-791198, suggesting that the higher abundances of alkylated PAHs correlate with a higher degree of aqueous alteration on the meteorite parent body of CM2 chondrites (Martins et al. 2015, Elsila et al. 2005).

In summary, the analysis of the abundances and distribution of the organic molecules present in meteorites helps to determine the physical and chemical conditions of the early solar system, and the prebiotic organic compounds available on the early Earth. 


\section{References}

Blanchard, I., Gounelle, M., Bourot-Denise, M., \& Kearsley, A. 2011, Meteorit. Planet. Sci., 46, A21.

Botta, O., Glavin, D. P., Kminek, G., \& Bada, J. L. 2002, OLEB, 32, 143-163.

Botta, O., Martins, Z., \& Ehrenfreund, P. 2007, Meteorit. Planet. Sci., 42: 81-92.

Bourot-Denise, M., Zanda, B., Marrocchi, Y., Greenwood, R. C., Pont, S., Hewins, R. H., Franchi, I. A., \& Cornen, G. 2010, abstract \#1533, 41st Lunar and Planetary Science Conference.

Browning, L. B., McSween, H. Y., \& Zolensky, M. E. 1996, Geochimica Cosmochimica Acta, 60, 2621-2633.

Burton, A. S., Glavin, D. P., Callahan, M. P., Dworkin, J. P., Jenniskens, P., \& Shaddad, M. H. 2011, Meteorit. Planet. Sci., 46, 1703-1712.

Caillet Komorowski, C., Boudouma, O., Renard, B., van de Moortele, B., \& El Goresy, A. 2011, Meteorit. Planet. Sci., 46, A35.

Chan, H.-S., Martins, Z., \& Sephton, M. A. 2012, Meteorit. Planet. Sci., 47, 1502-1516.

Chyba, C. F. \& Sagan, C. 1992, Nature, 355, 125-132.

Clayton, R. N. \& Mayeda, T. K. 1984, Earth Planet. Sci. Lett., 67, 151-161.

Cody, G. D. \& Alexander, C. M. O. D. 2005, Geochimica et Cosmochimica Acta, 69, 1085-1097.

Cooper, G. W. \& Cronin, J. R. 1995, Geochimica et Cosmochimica Acta, 59, 1003-1015.

Cournede, C., Gattacceca, J., Zanda, B., \& Rochette, P. 2011, Meteorit. Planet. Sci., 46, A50.

Cronin, J. R. \& Chang, S. 1993, In The Chemistry of Lifes Origin, edited by Greenberg J. M., Mendoza-Gomez C. X. and Pirronello, V. Dordrecht: Kluwer. pp. 209-258.

Ehrenfreund, P., Glavin, D. P., Botta, O., Cooper, G., \& Bada, J. L. 2001, PNAS, 98, 2138-2141.

Elsila, J. E., de Leon, N. P., Buseck, P. R., \& Zare, R. N. 2005, Geochimica et Cosmochimica Acta, 69, 1349-1357.

Glavin, D. P., Dworkin, J. P., Aubrey, A., Botta, O., Doty, J. H., Martins, Z., \& Bada, J. L. 2006, Meteorit. Planet. Sci., 41, 889-902.

Glavin, D. P., Aubrey, A. D., Callahan, M. P., Dworkin, J. P., Elsila, J. E., Parker, E. T., Bada, J. L., Jenniskens, P., \& Shaddad, M. H. 2010, Meteorit. Planet. Sci., 45, 1695-1709.

Glavin, D. P., Callahan, M. P., Dworkin, J. P., and Elsila, J. E. 2011, Meteorit. Planet. Sci., 45, 1948-1972.

Kimura, M., Grossman, J. N., \& Weisberg, M. K. 2011, Meteorit. Planet. Sci., 46, 431-442.

Martins, Z. 2011, Elements, 7, 3540.

Martins, Z. M. \& Sephton, A. 2009, In Hughes, A. B. (Ed.), Amino acids, peptides and proteins in organic chemistry. Wiley-VCH Verlag GmbH \& Co. KGaA, Weinheim, pp. 3-42.

Martins, Z., Watson, J. S., Sephton, M. A., Botta, O., Ehrenfreund, P., \& Gilmour, I. 2006, Meteorit. Planet. Sci., 41, 1073-1080.

Martins, Z., Alexander, C. M. O. D., Orzechowska, G. E., Fogel, M. L., \& Ehrenfreund, P. 2007, Meteorit. Planet. Sci., 42, 2125-2136.

Martins, Z., Modica, P., Zanda, B., \& Le Sergeant DHendecourt, L. 2015, Meteorit. Planet. Sci., 50, 926-943.

Merouane, S., Djouadi, Z., Brunetto, R., Borg, J., \& Dumas, P. 2011, Proceedings, EPSC-DPS Joint Meeting, pp.902.

Merouane, S., Djouadi, Z., Le Sergeant dHendecourt, L., Zanda, B., \& Borg, J. 2012, ApJ, 756, $154-160$.

Palmer, E. E. \& Lauretta, D. S. 2011, Meteorit. Planet. Sci., 46, 1587-1607.

Pizzarello, S., Huang, Y., \& Alexandre, M. R. 2008, PNAS, 105, 3700-3704.

Rodante, F., Marrosu, G., \& Catalani, G. 1992, Thermochimica Acta, 194, 197-213.

Rubin, A. E., Trigo-Rodriguez, J. M., Huber, H., \& Wasson, J. T. 2007, Geochimica et Cosmochimica Acta, 71, 2361-2382.

Schidlowski, M. 1988, Nature, 333, 313-318.

Schmitt-Kopplin, P. Gabelica, Z., Gougeon, R. D., Fekete, A., Kanawati, B., Harir, M., Gebefuegi, I., Eckel, G., \& Hertkorn, N. 2010, PNAS, 107, 2763-2768.

Schopf, J. W. 1993, Science, 260, 640-646.

Shimoyama, A. \& Ogasawara, R. 2002, OLEB, 32, 165-179. 
Tomeoka, K. \& Buseck, P. R. 1985, Geochimica et Cosmochimica Acta, 49, 2149-2163.

Zolensky, M. \& McSween, H. Y. 1988, In Meteorites and the early solar system, edited by Kerridge J. F., Matthews M. S. Tucson: University of Arizona Press. pp. 114-143.

Zolensky, M. E., Mittlefehldt, D. W., Lipschutz, M. E., Wang, M.-S., Clayton, R. N., Mayeda, T. K., Grady, M. M., Pillinger, C., \& Barber, D. 1997, Geochimica et Cosmochimica Acta, $61,5099-5115$. 